\section{Highlights from Recent Literature}

\section{Note:}

A more comprehensive list of literature and patents, updated quarterly, will be found in the searchable

Technical Database on the World Gold Council website, www.gold.org under Science and Industry domain.

\section{Analytical}

\subsection{Fabrication and Characterization of Gold Nanoparticles by Femtosecond Laser Ablation in an Aqueous Solution of Cyclodextrins}

Gold nanoparticles were produced by femtosecond laser ablation of a gold metal plate in an aqueous solution of $\alpha$-cyclodextrin (CD), $\beta .-\mathrm{CD}$, or $\delta .-\mathrm{CD}$. The gold nanoparticles exhibited the UV-vis absorption spectrum with a maximum absorption band at $520 \mathrm{~nm}$, similar to that of gold nanoparticles chemically prepared in a solution. A.V. Kabashin, M. Meunier, C. Kingston and J.H. Luong from the Laser Processing Laboratory, Department of Engineering Physics, Ecole Polytechnique de Montreal, Canada, Journal of Physical Chemistry B 2003, 107(19), 4527.

The size distribution of the nanoparticles measured by transmission electron microscopy (TEM) shifted to a drastically smaller size of approximately 2-2.4 $\mathrm{nm}$ and narrower size distribution of less than 1-1.5 nm at FWHM with an increase in the concentration of cyclodextrins. Both the particle size and size distribution were also dependent on the type of cyclodextrins used in aqueous solution. In particular, the gold colloids resulting from ablation in $10 \mathrm{mM}$ $\beta .-C D$ were conspicuously stable under aerobic conditions without any protective agent present. CDs formed an inclusion complex with ablated atoms to reduce the total concentration of embryonic nanoparticles formed in the plume CDs, as evident by Raman spectroscopy. The consecutive particle growth due to the mutual coalescence between nanoclusters and their neighbouring free gold atoms was severely limited in the presence of CDs.

\subsection{Size Distribution Analysis of Colloidal Gold by Small Angle X-Ray Scattering and Light Absorbance}

The data measured by small angle X-ray scattering (SAXS) and the extinction spectra observed by spectroscopy were examined as the in situ measurements of the size distribution of gold nanoparticles and were compared with the results obtained from the photographs by a transmission electron microscope (TEM). K. Nakamura, T. Kawabata, Y. Mori from the Department of Chemical Engineering and Materials Science, Doshisha University, Japan, Powder Technology 2003, 131(2-3), 120.

The mean size can be established from the wavelength of the peak absorbance using Mie theory. However, the particle size distribution can only be established from fitting the calculated spectrum to the experimental one of the sample with above $30 \mathrm{~nm}$ diameter. On the other hand, the scattering data measured by a SAXS instrument can est. the mean size from the slope of the Guinier plot, when the particle size distribution is narrow. In addition, the size distribution and mean size can be calculated by the experimental SAXS data when the log-normal or Schulz-Flory size distributions are assumed.

\subsection{Nanobubble Trouble on Gold Surfaces}

When analysing surfaces related to biosensors with in situ atomic force microscopy (AFM), the existence of nanobubbles called for attention. M. Holmberg, A. Kuehle, J. Garns, K. Morch and A. Boisen, Anja from the Danish Fundamental Metrology (DFM), Denmark, Langmuir 2003, 19(25), 10510. The bubbles seem to form spontaneously when gold surfaces are immersed in clean $\mathrm{H}_{2} \mathrm{O}$ and are probably a general phenomenon at $\mathrm{H}_{2} \mathrm{O}$-solid interfaces. Besides from giving rise to undesired effects in, for example, biosensors, nanobubbles can also cause artifacts in AFM imaging. The authors observed nanobubbles on unmodified gold surfaces, immersed in clean $\mathrm{H}_{2} \mathrm{O}$, using standard Si AFM probes. Nanobubbles can be made to disappear from contact mode AFM images and then to reappear by changing the scanning force. By combining contact mode AFM imaging and local force measurements, the interaction between the nanobubbles and the probe can be analyzed and give information about the characteristics of nanobubbles. A model of the forces between the AFM probe tip and the nanobubble indicates that a small tip cone angle and a relatively hydrophilic tip surface makes it possible to image nanobubbles with contact mode AFM even though the tip has penetrated the surface of the bubble. 


\section{Catalysis}

\subsection{Effects of Preparation Conditions on Gold/Y- Type Zeolite for CO Oxidation}

The effects of preparation conditions (i.e., chloroauric acid concentration, $\mathrm{pH}$ of gold solution, solution temperature, and mixing time) on gold supported on Y-type zeolite (Au/Y) for $\mathrm{CO}$ oxidation were studied by J. Lin and B. Wan, from the Department of Chemical Engineering, National Taiwan University, Taiwan, Applied Catalysis, B: Environmental 2003, 41(1-2), 83.

The catalysts were characterized by AA, temp.programmed reduction (TPR), XRD, TEM and XPS, in order to understand the correlation of the gold precursors in the solution and the gold particles in Y-type zeolite to the catalytic activity. It was found that more gold species prepared in the supercage of Y-type zeolite would enhance $\mathrm{Au} / \mathrm{Y}$ with higher catalytic activity. However, higher pH, higher gold concentration and lower temperature would generate more and larger gold species in the solution, which caused large gold particles prepared on the exterior surface of Au/Y. Due to the limited surface area, these large particles provided little activity for the reaction. Moreover, lower $\mathrm{pH}$ and lower gold concentration would generate more gold monomers in the solution at higher solution temperature, which caused more gold species prepared in the sodalite cage of Au/Y; due to molecule sieving effect, they were not available for $\mathrm{CO}$ oxidation. It is concluded that $\mathrm{Au} / \mathrm{Y}$ prepared from a gold solution with surface acidity modified Y-type zeolite $(1 \mathrm{~g}$ of zeolite per $125 \mathrm{~mL}$ soln.), with initial gold concentration around 1.46 .times. $10^{-3} \mathrm{M}$, solution $\mathrm{pH}$ 6, solution temperature at around $80^{\circ} \mathrm{C}$, and mixing time of $1 \mathrm{~h}$ would possess an optimum catalytic activity for $\mathrm{CO}$ oxidation.

\subsection{Catalytic Decomposition of Ozone on Gold- Containing $\mathrm{Au} / \mathrm{TiO}_{2}$ and $\mathrm{Au} / \mathrm{ZrO}_{2}$ Catalysts}

The catalytic decomposition of ozone on the gold-containing oxide catalysts $\mathrm{Au} / \mathrm{TiO}_{2}$ and $\mathrm{Au} / \mathrm{ZrO}_{2}$ was investigated in an isothermal flow reactor in the temperature range 2580.degree.C. P.M. Konova, A.I. Naydenov and D. Mehandjiev from the Institute of General and Inorganic Chemistry, Bulgarian Acad. of Science, Bulgaria, Bulgarian Chemical Communications 2002, 34(3/4), 437. The effect of increasing and decreasing the temperature during the catalytic process on the activity was studied. The activities of the two catalysts in the heterogeneous catalytic decomposition of ozone were compared and the conversion-time dependence was examined at different temperatures. The activation energies and rate constants of the heterogeneous catalytic decomposition of ozone were calculated for both catalysts. The higher activity in ozone decomposition observed with $\mathrm{Au} / \mathrm{TiO}_{2}$ is probably due to the difference in the chemical nature of the two supports.

\subsection{Nanosize Gold-Ceria Catalysts Promoted by Vanadia for Complete Benzene Oxidation}

New gold-vanadia-ceria catalysts for complete benzene oxidation were synthesized by D. Andreeva, R. Nedyalkova, L. Ilieva, M.V. Abrashev from the Institute of Catalysis, Bulgarian Academy of Sciences, Bulgaria, Applied Catalysis, A: General 2003, 246(1), 29. A high and stable catalytic activity was established. The influence of the preparation method and the vanadia loading on the catalytic activity was studied. The authors found that the presence of gold enhances the reducibility and the reactivity of VOx and ceria surface layers which results in the lowering of the reaction temperature The observed strong synergistic effect between gold and vanadia, when they present simultaneously on ceria, could be related to the specific interaction between gold, vanadia and ceria and the possibility to obtain nanosize gold and ceria particles, being able to produce more active oxygen species.

\subsection{Size and Support Effects for CO Adsorption on Gold Model Catalysts}

S.K. Shaikhutdinov, R. Meyer, M. Naschitzki M. Baeumer, H.J. Freund from the Department of Chemical Physics, FritzHaber-Institut der Max-Plank-Gesellschaft, Berlin, Germany, Catalysis Letters 2003, 86(4), 211 studied CO adsorption on gold particles deposited on well-ordered alumina and iron oxide films, with temperature-programmed desorption. Scanning tunneling microscopy was used to provide correlative structural characterization. The results show that the adsorption of $\mathrm{CO}$ on gold exhibits a size effect in that small particles adsorb CO more strongly. For a given particle size (apprx.3 nm), CO desorption temperature (at apprx.170 K) is essentially independent of the supports studied. Therefore, support effects seen in CO oxidation on real catalytic systems must arise from the interaction of oxygen rather than $\mathrm{CO}$ with these catalysts.

\subsection{Oxidation of $\mathrm{CO}$ on Gold Supported Catalysts Prepared by Laser Vaporization: Direct Evidence of Support Contribution}

Gamma.- $-\mathrm{Al}_{2} \mathrm{O}_{3}, \mathrm{ZrO}_{2}$, and $\mathrm{TiO}_{2}$ gold supported model catalysts have been synthesized by laser vaporization. Structural characterization using Transmission Electron Microscopy and XPS experiments have shown that the gold clusters deposited on the different supports have similar distribution of size centered around $3 \mathrm{~nm}$ and are in the metallic state. S. Arrii, F. Morfin, A.J. Renouprez and J.L. Rousset from the Institut de Recherches sur la Catalyse, Villeurbanne, France, Journal of the American Chemical Society 2004, 126(4), 1199. X-ray photoemission measurements also indicate lower binding energies than the usual Au $4 f 7 / 2$ at $84.0 \mathrm{eV}$ for both alumina and titania supported catalysts, indicating a modification of the 
electronic structure of the metal. The authors have taken benefit of these features to study the influence of the nature of the support toward $\mathrm{CO}$ oxidation activities without being hindered by particle size or gold oxidic species effects. By comparing the activities of the different catalysts, it is concluded that the nature of the support directly affects the activity of gold. The following tendency is observed: titania and zirconia are superior to alumina as supports, titania being slightly better than zirconia. From XPS and activity results the authors concluded that the existence of negatively charged clusters is not the key point to explain the high activity observed for $\mathrm{Au} / \mathrm{ZrO}_{2}$ and $\mathrm{Au} / \mathrm{TiO}_{2}$ catalysts and also that metallic gold is the major catalytically active phase. Hence, due to their very nature, titania and to a less extent zirconia should participate to the catalytic process.

\subsection{Catalytic Properties and Application of Gold Nanoparticles}

A review in Chinese by Q. Zhou and T. Shang from the Jiangsu Teachers' College of Technology, 213001, Peoples Republic of China, Huangjin 2003, 24(2), 6. The properties of gold nanoparticles arise from the special structures and effects of them. The high chemical activity and catalytic property of supported and un-supported gold nanoparticles lead to their application in chemical industry extensively. When gold is deposited as nanoparticles on metal oxides by means of precipitation or co-precipitation techniques, it exhibits surprisingly higher efficiency of catalytically oxidizing CO to form $\mathrm{CO}_{2}$ at room temperature than other precious metals. Gold supported on $\mathrm{TiO}_{2}$ can catalytically oxidizing propylene to form epoxy propane in the gas phase with selectivities above $90 \%$. Supported gold catalysts such as $\mathrm{Au} / \mathrm{Fe}_{2} \mathrm{O}_{3}$ and $\mathrm{Au} / \mathrm{ZrO}_{2}$ are applicable to the hydrogen production through low-temperature water-gas-shift reaction and the higher the crystallinity of the supports, the higher the catalytic activity of gold catalysts. A characteristic feature of gold catalysts in the hydrogenation of unsaturated hydrocarbon is that partial hydrogenation takes place very selectively. Unsupported and supported gold nanoparticles present great potentials as unique catalysts in liquid-phase reactions. All above topics have been reviewed in this paper.

\subsection{Electrocatalysis by Nanoparticles: Oxygen Reduction on Gold Nanoparticles- Electrodeposited Platinum Electrodes}

Oxygen reduction has been performed at gold nanoparticleelectrodeposited Pt electrodes (Au/Pt electrodes) in $\mathrm{O}_{2}$ saturated $0.1 \mathrm{M} \mathrm{KOH}$ solution. Cyclic voltammetry (CV) and rotating ring-disk electrode (RRDE) techniques have been used in this investigation by M. S. El-Deab and T. Ohsaka from the Interdisciplinary Graduate School of Science and Engineering, Department of Electronic Chemistry, Tokyo Institute of
Technology, Japan, Journal of Electroanalytical Chemistry 2003, 553,107 . Two reduction peaks were observed on the $\mathrm{CV}$ for the $\mathrm{O}_{2}$ reduction at the $\mathrm{Au} / \mathrm{Pt}$ electrodes. The reduction pathway of $\mathrm{O}_{2}$ to either $\mathrm{HO}_{2}$ - or $\mathrm{OH}$ - is highly dependent on the electrode potential and the gold surface coverage of the Pt electrode as well. The Au/Pt electrodes exhibit different electrocatalytic behaviour in three potential regions: At E>-300 mV, the 2electron reduction of $\mathrm{O}_{2}$ to $\mathrm{HO}_{2}$ predominates, at $-300>\mathrm{E}>-500$ $\mathrm{mV}$, the 4-electron reduction predominates, and at $\mathrm{E}<-500 \mathrm{mV}$, the 2-electron and 4-electron reduction pathways proceed concurrently. The maximum production of $\mathrm{HO}_{2}{ }^{-}$(as a major reduction product) was obtained at a potential of ca. $-240 \mathrm{mV}$ vs. $\mathrm{Ag}|\mathrm{AgCl}| \mathrm{KCl}(\mathrm{sat})$, which is more positive by ca. $250 \mathrm{mV}$ than the corresponding value at the bulk gold electrode (i.e. $500 \mathrm{mV}$ vs. Ag | $\mathrm{AgCl} \mid \mathrm{KCl}(\mathrm{sat}))$. The increase of the surface coverage of gold (i.e. increase of deposition time) on the Pt electrodes leads to a loss of this activity towards the $\mathrm{O}_{2}$ reduction to $\mathrm{HO}_{2}$. At a relatively higher negative potential, i.e. at $-400 \mathrm{mV}$, the 4-electron reduction of $\mathrm{O}_{2}$ to $\mathrm{OH}$ - occurs predominantly at all the examined Au/Pt electrodes with different gold loadings. Further negative increase of the potential (more neg. than $-400 \mathrm{mV}$ ) results in a partial contribution of the 2-electron reduction of $\mathrm{O}_{2}$ with the 4electron reduction. This behaviour could be understood in view of the potential dependence of the amount and the nature of the adsorbed species on the Au/Pt disk electrode surface.

\subsection{Surfactant-Protected Gold Particles: New Challenge for Gold-on-Carbon Catalysts}

$\mathrm{N}$-dodecyl-N,N-dimethyl-3-amino-1-propane sulfonate was used for stabilizing gold nanoparticles in water that afforded Au/C (SB-Au/C) when immobilized on activated carbon. S. Biella, F. Porta, L. Prati and M. Rossi from the Dipartimento di Chimica Inorganica Metallorganica e Analitica e Centro C.N.R. Universita di Milano, Milan, Italy, Catalysis Letters 2003, 90(12), 23. Characterization by XPS revealed a high atomic percentage of superficial gold (\% at. $\mathrm{Au}(4 \mathrm{f}) / \mathrm{C}(2 \mathrm{~s})$ ), compared with other $\mathrm{Au} / \mathrm{C}$ catalysts prepared by the immobilization of different sols. The activity of the SB-Au/C catalyst was found to be superior to that observed in the liquid-phase oxidation of ethylene glycol using other gold-on-carbon catalysts.

\subsection{Study on Preparation and Performance of Cobalt Oxide-Supported Nanometre Gold Catalysts}

Cobalt oxide supported nanometre gold catalysts were prepared by a co-precipitation and a organo-gold complex grafting methods. The catalytic performance for CO lowtemp. oxidation over these catalysts was evaluated and the effects of preparation methods and conditions on catalytic activities were investigated. It was found X. Zou, S. Qi, H. He, L. An and D. Duan from the Institute Applied Catalysis, Yantai 
Univ., Peoples Republic of China, Fenzi Cuihua 2003, 17(4), 264, that under the conditions controlled strictly, $3 \%$ $\mathrm{Au} / \mathrm{CO}_{3} \mathrm{O}_{4}$ catalysts prepared by the two methods mentioned above exhibit very high catalytic activities for $\mathrm{CO}$ oxidation and can oxidize $1 \%$ (vol.) $\mathrm{CO}$ in air to $\mathrm{CO}_{2}$ completely at $-22^{\circ} \mathrm{C}$ and $-5^{\circ} \mathrm{C}$ respectively. TEM and XRD measurements of the catalysts show that the size of gold particles on them is in the range of about ten nanometer, while the support is in a state of partially-crystalline $\mathrm{CO}_{3} \mathrm{O}_{4}$.

\section{Chemistry}

\subsection{Ramified Gold Deposits at The Gas Liquid Interface}

Ramified gold films have been prepared by electroless deposition at the surface of aqueous mixtures of hydrogen tetrachloroaurate and potassium chloride, covered by a positive charged dimethyldioctadecylammonium monolayer. R. Saliba, C. Mingotaud, F. Argoul and S. Ravaine from the Centre de Recherche Paul Pascal, Pessac, France, Journal of Electroanalytical Chemistry 2003, 544, 129. The authors show that increasing the concentration of chloride anions in the subphase induces a progressive modification of the morphology of the gold deposits, from compact to finely ramified patterns. Both the nature of the dissolving electrode and the presence of the positively charged surfactant monolayer have a profound effect on the structure and the growth mechanism of such ramified deposits.

\section{$4 \quad$ Electrochemistry}

\subsection{Quasi-Reversible Two-Electron Reduction of Oxygen at Gold Electrodes Modified with a Self-Assembled Submonolayer of Cysteine}

The electrochemical reduction of $\mathrm{O}_{2}$ was performed at gold electrodes modified with a submonolayer of a self-assembly (sub-SAM/Au) of a thiol compound (typically cysteine (CYST)) in $\mathrm{O}_{2}$-saturated 0.5M KOH, M.S. El-Deab, T. Ohsaka, from the Interdisciplinary Graduate School of Science and Engineering, Department of Electronic Chemistry, Tokyo Institute of Technology, Japan, Electrochemistry Communications 2003, 5(3), 214. At bare gold electrode $\mathrm{O}_{2}$ reduction reaction proceeds irreversibly, while this reaction is totally hindered at gold electrodes with a compact structure of CYST over its surface. The partial reductive desorption of the compact CYST monolayer was achieved by controlling the potential of the CYST/gold electrode, giving a submonolayer coverage of the thiol compound over the gold electrode surface (subSAM/Au), at which the CYST molecules selectively block the gold(100) and gold(110) fractions (the so-called rough domains) of the polycrystalline gold while the gold(111) component (the so-called smooth domains) remains bare (i.e., uncovered with CYST). This sub-SAM/gold electrode extraordinarily exhibits a quasi-reversible 2-electron reduction of $\mathrm{O}_{2}$ in alkaline medium with a peak seperation (.DELTA.Ep) between the cathodic and anodic peak potentials (Epc,Epa) of approximately $60 \mathrm{mV}$. The ratio of the anodic current to the cathodic one is close to unity. The formal potential (Eo') of this reaction is found to equal $-150 \mathrm{mV}$ vs. $\mathrm{Ag} / \mathrm{AgCl} / \mathrm{KCl}$ (sat.).

\subsection{Electrochemical Resolution of 15 Oxidation States for Monolayer Protected Gold Nanoparticles}

The first observation of 15 voltammetric quantized charging peaks for a solution of hexanethiol-capped gold nanoparticles (so-called monolayer protected clusters MPCs) at room temperature is reported by B.M. Quinn, P. Liljeroth, V. Ruiz, T. Laaksonen and K. Kontturi from the Laboratory of Physical Chemistry and Electrochemistry, Helsinki University of Technology, Finland, Journal of the American Chemical Society 2003, 125(22), 6644, where the variation in peak spacing with increasing charge stored in the metal core is discussed in terms of MPC capacitance.

\subsection{Anodic Oxidation of Free Nitric Oxide at Gold Electrodes Modified by a Film of Trans- $\left(\mathrm{Ru}(\mathrm{III})\left(\mathrm{NH}_{3}\right)_{4}\left(\mathrm{SO}_{4}\right)_{4} \mathrm{pic}\right]^{+}$and Molybdenum Oxide}

Gold surfaces were modified with an electrochemical deposited layer of non-stoichiometric molybdenum oxides. V. Mori, J.C. Toledo, H.A.S. Silva, D.W. Franco, M. Bertotti, Instituto de Quimica, Department of Chemistry, Universidade de Sao Paulo, Brazil, Journal of Electroanalytical Chemistry 2003, 547(1), 9. At these surfaces, trans$\left(\mathrm{Ru}(\mathrm{III})\left(\mathrm{NH}_{3}\right)_{4}\left(\mathrm{SO}_{4}\right)_{4} \mathrm{pic}\right]+$ complex was incorporated in a controlled way by cycling the potential consecutively in the range +0.50 to $-0.25 \mathrm{~V}$ at $\mathrm{pH}$ 2.6. Very reproducible voltammetric curves corresponding to the electrochemical process of the ruthenium complex were obtained, confirming the immobilization of the material into the molybdenum oxide film. The anodic oxidation of nitric oxide (NO) at pH 7.4 was investigated at the modified electrode containing the molybdenum oxide+trans-( $\left.\mathrm{Ru}(\mathrm{III})\left(\mathrm{NH}_{3}\right)_{4}\left(\mathrm{SO}_{4}\right)_{4} \mathrm{pic}\right]+$ complex and an enhancement in the current response was observed compared with the signal at a bare electrode. The rate for NO electrochemical oxidation was dependent on the amount of catalytic ruthenium sites dispersed into the molybdenum oxide film. A linear relationship between current signals measured by square wave voltammetry and NO concentration was obtained in the 0-10 $\mu \mathrm{m}$ range. The applicability of the modified electrode as a sensor for real-time NO monitoring was also demonstrated. 


\subsection{Cyclic Voltammetry and Scanning \\ Electrochemical Microscopy of Ferrocenemethanol at Monolayer and Bilayer- Modified Gold Electrodes}

Ferrocenemethanol was chosen to study quantitatively the kinetics of the heterogeneous electron transfer at mono- and bilayer-modified gold electrodes. C. Cannes, F. Kanoufi, A.J. Bard from the Department of Chemistry and Biochemistry, The University of Texas USA, Journal of Electroanalytical Chemistry 2003, 547(1), 83. The monolayer was prepared by tetradecanethiol adsorption on the gold surface and the bilayer was formed by phospholipid adsorption on the first layer. The apparent electron transfer rate constants at the modified gold electrodes were measured by cyclic voltammetry (Tafel anal.) and from scanning electrochemical microscopy approach curves, as well as by simulations of the cyclic voltammograms. The values obtained by simulation based on a CEC mechanism were close to the values obtained by both other techniques. The results show that the surface coverage was high and depended on the thickness of the layer (0.993 for the monolayer and 0.9998 for the bilayer). The major consequence of this high coverage is a large decrease in the apparent rate constant for ferrocenemethanol oxidation that depended on the thickness of the modifying layer. The results also indicate that ferrocenemethanol could permeate to a small extent into the monolayer. The electron transfer at the bilayer is mainly governed by diffusion of the mediator through pinholes with electron transfer occurring at the free sites on the electrode rather than permeation.

\subsection{Influence of a Nanoscale Gold Thin Layer on $\mathrm{Ti} / \mathrm{SnO}_{2}-\mathrm{Sb}_{2} \mathrm{O}_{5}$ Electrodes}

A nanoscale gold thin film and its influence on $\mathrm{Ti} / \mathrm{SnO}_{2}-\mathrm{Sb}_{2} \mathrm{O}_{5}$ coating were studied by SEM and electrochemical methods such as cyclic voltammetry, electrochemical impedance spectroscopy (EIS), and the chronopotentiometric technique in both acidic and caustic solutions by A. Chen and S. Nigro from the Department of Chemistry, Lakehead University, Thunder Bay, Canada Journal of Physical Chemistry B 2003, 107(48), 13341. The gold thin film was fabricated by sputtering, and its thickness was established using electrochemical methods. The nanoscale gold thin film on the Ti substrate exhibits electrochemical behaviour similar to that of a polycrystalline gold electrode. The presence of a nanoscale gold thin film on the Ti substrate can effectively prevent the growth of a $\mathrm{TiO}_{2}$ insulating layer between the substrate and the $\mathrm{SnO}_{2}-\mathrm{Sb}_{2} \mathrm{O}_{5}$ coating, resulting either from the thermal procedure during the fabrication of the oxide electrodes or from the electrochemical process during the lifetime tests, thus greatly prolonging the service lifetime of the $\mathrm{SnO}_{2}-\mathrm{Sb}_{2} \mathrm{O}_{5}$ electrodes. The failure mechanisms of the $\mathrm{SnO}_{2}-\mathrm{Sb}_{2} \mathrm{O}_{5}$ electrodes also were studied using the EIS and SEM techniques.

\subsection{Oxygen Reduction at Platinum Modified Gold Electrodes}

The reduction of oxygen was studied on polycrystalline gold electrodes modified by Pt deposited spontaneously from an aqueous $\mathrm{K}_{2} \mathrm{PtCl}_{6}$ solution via the displacement of copper or lead adlayers. M.Van Brussel, G. Kokkinidis, A. Hubin and C. Buess-Herman from the Service de Chimie Analytique et Chimie des Interfaces, Faculte de Sciences, Universite Libre de Bruxelles, Brussels, Electrochimica Acta 2003, 48(25-26), 3909.

The change in the surface composition and morphology was checked by XPS, AES and AFM data. The kinetic results showed that such electrodes may present a higher catalytic activity compared to bulk Pt electrodes during a scan where the potential is made more positive and is thus clearly expressed by an hysteresis in the CV curves. The displacement of copper and lead deposits gave similar amplitudes of the hysteresis but the modified electrodes obtained via a lead deposit present a better stability upon cycling in acid solutions. The observed behaviour can be correlated to the modification of the $\mathrm{M}-\mathrm{OH}$ formation and reduction on noble metals.

\section{$5 \quad$ Electronics and Sensors}

\subsection{Organic Molecular Films on Gold Versus Conducting Polymer: Influence of Injection Barrier Height and Morphology on Current- Voltage Characteristics}

The current-voltage characteristics I(V) of model organic devices are studied under ultra-high-vacuum conditions by $\mathrm{N}$. Koch, A. Elschner, J. Schwartz and A. Kahn from the Department of Electrical Engineering and Department of Chemistry, Princeton University, USA Applied Physics Letters 2003, 82(14), 2281. Active materials are N,N'-bis-(1naphthyl)-N,N'-diphenyl-1,1-biphenyl-4,4'-diamine (.alpha.NPD) and pentacene, electrode materials are polycrystalline gold and the conductive polymer poly(3,4ethylenedioxythiophene)/poly(styrenesulfonate) (PEDOT/PSS). Despite a similar work function of electrode material surfaces (apprx.5 eV), hole injection from PEDOT/PSS is significantly more efficient than from gold, due to a smaller hole injection barrier. Hole injection characteristics from gold electrodes for devices made from $\alpha$-NPD are independent of deposition sequence and substrate used. Pentacene devices exhibit serious asymmetries in that respect. These are caused by a strong dependence of morphololgy and preferred molecule orientation on the substrate for the crystalline material. 


\subsection{Physico-Chemical Contribution of Gold Metallic Particles to the Action of Oxygen on Tin Dioxide Sensors}

The role of a metal (gold) on the electrical response on tin oxide sensor was studied thanks to the development of a particular test bench: it allows the separation of the atmosphere surrounding the $\mathrm{SnO}_{2}$ region in contact with gold electrode from the atmosphere in the area between electrodes. P. Montmeat, J.C. Marchand, R. Lalauze, J.P. Viricelle, G. Tournier and C. Pijolat from the Centre SPIN, Ecole Nationale Superieure des Mines, France, Sensors and Actuators, B: Chemical 2003, B95(1-3), 83. The action of oxygen and thus of a reducing gas such as $\mathrm{CO}$ was investigated and is greatly enhanced in the region containing gold. This result in conjunction with calorimetric tests indicates the creation of specific oxygen species at the metaloxide interface. A qualitative physicochemical model based on the electronic effect of these adsorbed species, resulting in the increase of space charge area is discussed.

\section{$6 \quad$ Medical and Dental}

\subsection{Gold Concentration in Blood in Relation to the Number of Gold Restorations and Contact Allergy to Gold}

Previous studies have demonstrated an association between gold allergy and the presence of dental gold restorations. The aim of the present study, I. Ahnlide, C. Ahlgren, B. Bjoerkner, M. Bruze, T. Lundh, H. Moeller, K. Nilner and A. Schuetz, from the Department of Occupational and Environmental Dermatology, Malmoe University Hospital, Sweden, Acta Odontologica Scandinavica 2002, 60(5), 301, was to investigate the relationship between the concentration of gold in blood (B-Au) and the number of tooth surfaces with gold alloys in subjects with and without contact allergy to gold. In 80 patients referred for patch testing because of eczematous disease, blood samples were taken and analyzed for B-Au using inductively coupled plasma mass spectrometry.

The detection limit for gold detection was 0.04 .mu.g/L. In addition, a dentist made a clinical and radiological examination of the patients and registered the number of dental gold surfaces. Patients with dental gold restorations had a statistically significantly higher B-Au in Mann-Whitney $U$ test ( $P=$ 0.025), (range $<0.04-1.07 \mu \mathrm{m} \mathrm{g} / \mathrm{L}$ ) than patients without (range $<0.04-0.15 \mu \mathrm{m} \mathrm{g} / \mathrm{L}$ ). Furthermore, a positive correlation was found between B-Au and the number of dental gold surfaces $(P<0.01)$. There was no statistically significant difference in B-Au between persons with and without contact allergy to gold. The study thus indicates that gold is released from dental restorations and taken up into the circulation.

\subsection{Sintering Inlay. Formation of Surface Alloy by using Art Clay Silver and Gold Plate}

Metal with excellent physical properties as a restoration material usually requires a casting process. A minimum of five to six hours is required to complete this casting process. Furthermore, this requires skill in controlling casting timing and adjusting casting shrinkage. The authors, K. Ohkuma and T. Yoneyama from the School of Dentistry at Tokyo, The Nippon Dental University, Japan, Journal of Japanese Society for Dental Products 2002, 16(1), 3, have devised a production method that creates metal inlays without a casting process. Art clay silver is sintered in a microwave oven, and then gold is alloyed onto the surface with a laser beam to improve color stability. If it is able to make this type of metal inlay in thirty minutes, it is possible to finish treatment in a one-day session.

The authors performed the following tests and experiments: (1) sintering art clay silver with various heating times; (2) a hardness test of the gold alloy surface; and (3) a color stability test. A Vickers Hardness of 34 was obtained by sintering art clay silver for four minutes in a microwave oven. The hardness of the surface gold alloy, when gold sheets of 50 .mu.m and 100 .mu.m are used, is Vickers Hardness 44.8 - 57.0 and 51.7 - 69.8 respectively. After soaking surface gold alloys (pressure welded sheets of 50 .mu.m and 100 .mu.m) in $0.1 \%$ solution of sodium sulfide at 37.degree.C for 3 days, their $L^{*}$ are 71.5 and 73.5 respectively., and they meet specifications (T6105: L* greater than 70) set for gold-silverpalladium alloys for casting. The LAMBDA. $L^{*}$, difference in lightness before and after the soaking of the surface gold alloy is reduced to $2.2-2.8$ or $1 / 10$ of the sintered silver. Using gold alloy to create the surface of the silver inlay improves its color stability. Utilizing a microwave oven and a laser system, metal inlays can be made in thirty minutes.

\section{$7 \quad$ Metallurgy, Materials and Coatings}

\subsection{Synthesis and Characterization of Nanoporous Gold Nanowires}

C. Ji, and P. Searson, from the Department of Materials Science and Engineering, Johns Hopkins University, Baltimore, USA, Journal of Physical Chemistry B (2003), 107(19), 4494, report on a 2-step process for the fabrication of nanoporous nanowires. The fabrication process involves electrochemical deposition of a single-phase binary alloy into a nanoporous template, followed by subsequent etching of the less noble component. These particles are characterized by diameters of $20 \mathrm{~nm}$ or larger, lengths of up to tens of microns, and a porous structure with controlled pore dimensions. In contrast to conventional solid nanoparticles, nonporous nanowires have a high surface area and a well-defined pore morphology 
Also, nanoporous segments can be incorporated into multisegment nanorods or nanowires. Here, the authors demonstrate the fabrication of nanoporous $\mathrm{Au}$ (NPG) nanowires and multisegment nanowires incorporating Au/NPG layers and Pt/NPG layers.

\subsection{Anelasticity Study on Motions of Atoms in the Grain Boundary Regions in Nanocrystalline Gold}

High-d. nanocrystalline (n-) gold was prepared by the gas deposition method by $H$. Tanimoto, S. Sakai, and $H$. Mizubayashi, from the Institute of Materials Science, University of Tsukuba, Tsukuba, 305-8573, Japan, Materials Transactions 2003, 44(1), 53. Various anelastic and plastic creep processes associated with the grain boundary (GB) regions were observed. The vibrating reed measurements at $102 \mathrm{~Hz}$ with strain amplitude of $10^{-6}$ show a very broad internal friction peak near $95 \mathrm{~K}$, Qp.95 K-1, and a steep increase in the anelastic strain above $200 \mathrm{~K}$, .epsilon.a-I, >200 K. The tensile tests show a steep increase in the anelastic strain above 200 K, .epsilon.a-II, >200 K, for the stress beyond a few MPa and a linear plastic creep strain above $200 \mathrm{~K}$, .epsilon.pc-I, for stress range between $30 \mathrm{MPa}$ and $150 \mathrm{MPa}$. The activation parameters, 1/.tau.0 of 3 .times. $1011 \mathrm{~s}-1$ and $\mathrm{E}$ of $0.16 \mathrm{eV}$, are found for Qp.95 K-1, where .tau.0 and E are a pre-exponential factor and an activation energy of the relaxation time .tau.. The authors surmised that simple relaxation processes are responsible for Qp.95 K-1. The values of 1/.tau.0 and E found for .epsilon.a-f, >200 K and .epsilon.a-II, >200 K decrease with increasing the applied stress or the temp., indicating that their atomic processes are the same feather. Further, E found for .epsilon.pc-1 is similar to or slightly smaller than that of .epsilon.a-II, $>200 \mathrm{~K}$. These observations indicate that the atomic motions in the $\mathrm{CB}$ regions of .eta.-gold develop in scale in the order of the underlying processes for .epsilon.a-l, $>200 \mathrm{~K}$, epsilon.a-II, >200 K and .epsilon.pc-1, and are so different from those in the conventional polycryst. gold.

\subsection{A Composite Gold-Silicon Oxide Surface for Mesoscopic Patterning}

A composite surface comprising evenly distributed Si oxide islands on a gold substrate is described by K. Shabtai, S.R. Cohen, $\mathrm{H}$. Cohen and I. Rubinstein from the Department of Materials and Interfaces and Chemical Services Department, Weizmann Institute of Science, Rehovot, Israel, Journal of Physical Chemistry B 2003, 107(23), 5540. The composite surface was prepared by evaporation of a thin $(50 \mathrm{~nm})$ gold layer on oxide-free ( $\mathrm{H}$-passivated) $\mathrm{Si}$, followed by thermal diffusion of Si through the gold layer, gradually forming islands of $\mathrm{SiO}_{2}$ on the gold surface. The rate of $\mathrm{Si}$ diffusion through the gold, and hence, the rate of $\mathrm{SiO}_{2}$ island formation, is controlled by the annealing temperature, the Si crystallography face, the gold film thickness, and the contacting atmosphere. The Au$\mathrm{SiO}_{2}$ composite surfaces can be used in applications requiring substrates patterned on a mesoscopic scale, while exposing two chemically dissimilar phases. One such application is shown here, namely, the formation of thiol-silane monolayers, for which the distribution of the different molecules in the resultant monolayer is detected by the substrate composition. The XPS controlled surface charging (CSC) method was used to establish a site-selective adsorption. The $\mathrm{SiO}_{2}$ islands are rather labile, shifting and aggregating upon self-assembly of alkanethiol molecules on the gold exposed areas. Pretreatment of the islands with a long-chain silane stabilizes the morphology.

\subsection{Flip Chip Connections using Gold Stud Bumps}

As power requirements and operating frequencies increase, more and more designs will look toward ball bumps as an interconnect solution. While solder has traditionally been the incumbent material for these bumps, solder's limitations have become manufacturing and performance limitations. As a result, packaging designers are looking toward gold bumps as a strong contender in the first-level interconnect battle. This paper by J. Jordan from Palomar Technologies, Inc., USA, International SAMPE Symposium and Exhibition 2003, 48 (Advancing Materials in the Global Economy-Applications, Emerging Markets and Evolving Technologies, Book 1), 495 will briefly discuss the limitations of the solder connection process and compare that to the gold bump process. Furthermore, it will describe the four leading alternatives for achieving the gold bump flip-chip connection.

\subsection{Purplish Gold-Colored Noble Metal Ornamental Materials, their Manufacture, and their use as Ornaments and Accessories}

Ornamental purplish gold-colored noble metals with their entire or partial surfaces comprising 70-85 wt.\% Au-balance Al alloys are claimed by S. Yamazaki, N. Ishii and K. Sawada from the Ishifuku Metal Industry Co., Ltd., Japan JP 2003183710, A2, July 2003. The alloys may be reinforced with $\mathrm{Au}, \mathrm{Ag}$, Pt, or their base alloys by diffusion bonding. An Au-Al alloy ingot, prepared by vacuum melting, is treated by centrifugal atomization to obtain spherical alloy particles that are packed in a mold and discharge sintered to give purplish gold-colored ornamental materials. Noble metal reinforcement sheets may also be added before sintering. Ornaments and accessories, e.g. earrings, tie pins, cuff links, necklaces, watch faces, manufactured by machining and polishing of the alloys are also claimed. The alloys have enough strength for use as ornaments and accessories. 


\section{$8 \quad$ Nanotechnology}

\subsection{Shape Controls of Electrochemically Prepared Gold Nanorods}

Gold nanorods were prepared in aqueous micelles with ionic surfactants composed of the supporting electrolyte surfactant (hexadecyltrimethylammonium bromide) and a shape-inducing cosurfactant by an electrochemical method by T. Onishi; H. Yao, S. Sato and K. Kimura, from the Department of Material Science, Faculty of Science, Himeji Institute of Technology, Kamigori-cho, Ako-gun, Japan Transactions of the Materials Research Society of Japan 2002, 27(1), 159. During the syntheses, a gold plate as the anode is converted sacrificially to form nanorods. The authors examined the effects of cosurfactants and/or silver ion release from the immersed silver plate on aspect ratios of the obtained nanorods. Cosurfactants play a crucial role for detecting the shapes of the rods. By controlling these factors, the gold nanorods are grown to be submicrometers in length with a very high aspect ratio.

\subsection{Ultrafast Dynamics of Gold-Based Nanocomposite Materials}

Ultrafast electron dynamics are compared for small (2.5-4.0 $\mathrm{nm}$ average diameter) gold nanoparticles in two different surrounding environments. J.S. Melinger, V.D. Kleiman, D. McMorrow, F. Groehn, B.J. Bauer and E. Amis from the Electronics Science and Technology Division, Naval Research Laboratory, Washington,USA Journal of Physical Chemistry A 2003, 107(18), 3424. In one case, an aqueous solution contains gold nanoparticles (with average diameters of either 2.5 or $4.0 \mathrm{~nm}$ ) embedded inside polyamidoamine (PAMAM) dendrimers. In the other case, hexanethiol-passivated (HT) gold nanoparticles, with an average diameter of $3.6 \mathrm{~nm}$, are suspended in $\mathrm{CH}_{2} \mathrm{Cl}_{2}$ solution. Two-color fs pump-probe spectroscopy is used to excite and probe the dynamics of the gold nanoparticles in the region of the surface plasmon resonance. The transient response is measured as a function of laser excitation fluence $\left(\mathrm{J} / \mathrm{cm}^{2}\right)$. For the metal-dendrimer nanocomposites, the transient response consists of a singleexponential decay that relaxes with a time const. of $<1.2$ ps and is due mainly to electron-phonon coupling. The relaxation time scale shows a weak dependence on excitation fluence but is essentially independent of the size of the gold nanoparticle embedded inside the dendrimer (for the size range studied). In contrast to the metal-dendrimer systems, the transient response of the Au- $\mathrm{HT}$ in $\mathrm{CH}_{2} \mathrm{Cl}_{2}$ reveals both electron-phonon and phonon-phonon relaxation components, and the relaxation time scale shows a relatively stronger dependence on excitation fluence. A qualitative discussion is given for the different relaxation characteristics that are observed for the two gold nanoparticle systems.

\subsection{Preparation and Organization of Nanoscale Polyelectrolyte-Coated Gold Nanoparticles}

The layer-by-layer (LbL) deposition of oppositely charged polyelectrolytes from adsorption solutions of different ionic strength onto .approximately7 nm diameter carboxylic acidderivatized gold nanoparticles was studied by K.S. Mayya, B. Schoeler and F. Caruso from the Max Planck Institute of Colloids and Interfaces, Potsdam, Germany, Advanced Functional Materials (2003), 13(3), 183. The polyelectrolytemodified nanoparticles were characterized by UV-visible spectrophotometry, microelectrophoresis, analytical ultracentrifugation, and TEM. UV-visible data showed that the peak plasmon absorption wavelength of the gold nanoparticles red shifted after each adsorption step, and microelectrophoresis experiments revealed a reversal in the surface charge of the nanoparticles following deposition of each layer.

These data are consistent with the formation of polyelectrolyte layers on the nanoparticles. Analytical ultracentrifugation showed an increase in mean nanoparticle diameter on adsorption of the polyelectrolytes, confirming the formation of gold- core/polyelectrolyte-shell nanoparticles. TEM studies showed no signs of aggregation of the polyelectrolyte-coated nanoparticles. The adsorption of the polyelectrolyte-coated gold nanoparticles onto oppositely charged planar supports also was examined UVvisible spectrophotometry and atomic force microscopy showed increased amounts of nanoparticles were adsorbed with increasing ionic strength of the nanoparticle dispersions. This allows control of the nanoparticle surface loading by varying the salt content in the nanoparticle dispersions used for adsorption. The LbL strategy used in this work is expected to be applicable to other nanoparticles (e.g., semiconductors, phosphors), thus providing a facile means for their controlled surface modification through polyelectrolyte nanolayering. Such nanoparticles are envisaged to have applications in the biomedical and bioanalytical fields, and to be useful building blocks for the creation of advanced nanoparticle-based films.

\subsection{Nanometer-Sized Gold Particles Supported on $\mathrm{SiO}_{2}$ by Deposition of Gold Sols from $\mathrm{Au}\left(\mathrm{PPh}_{3}\right)_{3} \mathrm{Cl}$}

A gold $/ \mathrm{SiO}_{2}$ system with most of the metal particles less than $2.0 \mathrm{~nm}$ in size were prepared by deposition on silica of preformed gold sols derived from $\mathrm{Au}\left(\mathrm{PPh}_{3}\right)_{3} \mathrm{Cl}$. G. Martra, L. Prati, C. Manfredotti, S. Biella, M. Rossi and S. Coluccia, from the Department of Chemistry I.F.M., Universita di Torino, Turin, Italy, Journal of Physical Chemistry B 2003, 107(23), 5453. In this form, it was inactive in the $\mathrm{CO}+\mathrm{O}_{2}$ reaction at ca. $333 \mathrm{~K}$, because of the presence of phosphine ligands on the surface of metal particles. The system became 
catalytically active in this reaction after treatment in $\mathrm{O}_{2}$ at $673 \mathrm{~K}$, which also resulted in a slight sintering of the metal phase (mean size $=2.9 \mathrm{~nm}$ ). By subsequent heating in $\mathrm{H}_{2}$ at $673 \mathrm{~K}$, the mean size of gold particles increased up to 3.4 $\mathrm{nm}$. Such particles exhibited a lower amount of step surface sites able to adsorb CO, but became more active in the CO + $\mathrm{O}_{2}$ reaction. This suggested that the reaction steps involving oxygen can occur more effectively on gold sites present on larger and smoother gold particles.

\subsection{Microwave Heating for the Preparation of Nanometer Gold Particles}

A feasible method to synthesize gold nanoparticles with uniform size by microwave heating is reported by F.K. Liu, C.J. Ker, Y.C. Chang, F.H. Ko, T.C. Chu and B.T. Dai from the National Nano Device Lab., Hsinchu, Taiwan, Japanese Journal of Applied Physics, Part 1: Regular Papers, Short Notes \& Review Papers 2003, 42(6B), 4152. A closed chamber of a microwave system with precise temperature control function was employed to synthesize the gold nanoparticles. The data demonstrates that the temperature plays an important role in the synthesis of gold nanoparticles. With higher synthesis temperature, longer heating time duration and rapid gradient of temperature increase, the smaller diameter and the narrow size distribution of the gold nanoparticles were obtained. The concentrations of citrate represent the reductant in the synthesis reaction, which also influence the morphology of gold nanoparticles. The data demonstrates that gold nanoparticles with highly reproducible sizes could be synthesized by microwave heating.

\subsection{Optical Properties and Device Applications of Polymer Films Containing Gold or Silver Nanoparticles}

Gold or silver nanoparticles have characteristic absorption bands in the UV-near IR region due to surface plasmon oscillation of free electrons. Thus, they show clear colors such as blue and red. Spectroscopic properties of those nanoparticles depend on the morphology of the single particle as well as the agglomerates. Recently, various methods for the preparation of nanoparticle-doped thin films have been developed, and their optical applications have been increasing interests. In this review article by Y. Niidome and S.Yamada from the Department of Applied Chemistry, Graduate School of Engineering, Kyushu University, Fukuoka, Japan, Nippon Shashin Gakkaishi 2003, 66(4), 349, the preparation and dichroic properties of gold- or silvernanoparticle-doped films are described, together with some photonics applications.

\subsection{Luminescent Blinking of Gold Nanoparticles}

Spin-coated gold colloids showed a time-dependent increase in emission upon illumination at $532 \mathrm{~nm}$. The emission originated from point-like locations and displayed blinking. C.D. Geddes, A. Parfenov, I. Gryczynski and J.R. Lakowicz from the Department of Biochemistry and Molecular Biology, Center for Fluorescence Spectroscopy, University of Maryland at Baltimore, Baltimore, USA, Chemical Physics Letters 2003, $\mathbf{3 8 0}(\mathbf{3}, \mathbf{4}), \mathbf{2 6 9}$. The emission spectra of the individual spots and their spatial location remained constant which is distinct from recent reports on silver nanoparticles. These results suggest the use of emission from gold clusters in nanophotonics devices and as luminescent probes with potential applications in medical imaging.

\subsection{Synthesis, Characterization and Properties of Water-Soluble Gold Nanoparticles with tunable Core Size}

Robust, water-soluble gold clusters protected by monolayers of ligands containing a short alkyl chain (C7) close to the gold surface and a triethylene glycol monomethylether unit (TEG) to impart solyation in water and other polar solvents were prepared and characterized. P. Pengo, S. Polizzi, M. Battagliarin, L. Pasquato and P. Scrimin from the Dipartimento di Chimica Organica, Universita di Padova, Italy, Journal of Materials Chemistry 2003, 13(10), 2471. Thiol 7 (N1 - \{2-(2-(2-methoxyethoxy) ethoxy] ethyl \}-8sulfanyloctanamide) constitutes a good and versatile capping agent for the preparation of these nanoparticles. By tuning the Au/thiol ratio and sodium borohydride addition rate, nanoparticles with different core diameters ranging from 1.5 to $4.2 \mathrm{~nm}$, as detected by TEM analysis, could be obtained. The size distribution of the gold cores appears to become broader as the Au/thiol ratio used to prepare the nanoparticles increases. Characterization of these nanoclusters also by NMR, UV-Vis and FTIR spectroscopies is reported. Solyation properties have been studied in a large variety of solvents and different solyation behaviours were observed for nanoparticles of different sizes. Exchange reactions were carried out successfully with small $(1.9 \mathrm{~nm})$ and large nanoparticles $(4.2 \mathrm{~nm})$ using dodecanethiol as the entering thiol. This demonstrates that these materials can be used for the preparation of nanoclusters with different functional groups sol. in polar solvents including water. The synthetic procedure described represents a facile route to tailoring the size and solyation properties of gold nanoparticles. 
8.9 Synthesis and Characterization of Gold/Polypyrrole Core-Shell Nanocomposites and Elemental Gold Nanoparticles based on the Gold-Containing Nanocomplexes prepared by Electrochemical Methods in Aqueous Solutions

The Authors Y-C. Liu and T. Chuang from the Department of Chemical Engineering and Polymer Material RD Center, Van Nung Institute of Technology, Peoples Republic of China, Journal of Physical Chemistry B 2003, 107(45), 12383, report here the first electrochemical pathway to prepare goldcontaining nanocomplexes with the mean diameter of $2 \mathrm{~nm}$ in $0.1 \mathrm{~N} \mathrm{KCl}$ aqueous solutions without addition of any stabilizer. Encouragingly, polypyrrole-(PPy-) coated gold nanocomposites with a core-shell structure and a diameter smaller than $8 \mathrm{~nm}$ can be prepared by the formation of selfassembled monolayers and further orderly autopolymn. of pyrrole monomers on these nanocomplexes. The prepared Au/PPy nanocomposites should exhibit extremely high cond., as indicated by the analyses of X-ray diffraction (XRD), XPS, and surface-enhanced Raman scattering (SERS). Meanwhile, elemental $\mathrm{Au}(0)$ nanoparticles of a mean diameter of $5 \mathrm{~nm}$ can be obtained by treating the Au/PPy-nanocompositescontaining solution with sonication.

\section{$9 \quad$ Refining}

\subsection{Ultrasonic Elution of Gold from Activated Carbon}

Gold cyanide loaded in activated carbon was eluted with or without the applied ultrasound at 70 or 90 .degree., using a lab.-scale elution column immersed in an ultrasonic bath and applying 3 elution systems. D. Feng, H. Tan and J.S.J. van Deventer from the Department of Chemical Engineering, The University of Melbourne, Australia Minerals Engineering 2003, 16(3), 257. The rate of elution was increased by applying the ultrasound treatment, and by addition of EtOH to the aqueous elution system. The gold recovery from the plantloaded carbon bed was $97.6-99.9 \%$ by the chemical elution with ultrasound, or 57.2-59.5\% without the ultrasound, using aqueous $\mathrm{KCN}-\mathrm{NaOH}$ or $\mathrm{Na}_{2} \mathrm{CO}_{3}$ solutions at 21 bed vols. The similar gold elution with deionized water was 91.4 or $48 \%$ respectively. Comparable results were obtained for the associated elution of silver and Nickel. The introduction of ultrasound in the conventional elution systems increased the gold recovery from activated carbon.

\subsection{On-Site Gold Refining of Cyanide Liquors by Solvent Extraction}

The refining of gold in crude cyanide solutions from ore leaching was evaluated by selective extraction in the presence of silver and Copper ions, especially at remote oreleaching sites. M.D. Adams from Lakefield Oretest, Malaga Australia, Minerals Engineering 2003, 16(4), 369. The gold is selectively extracted into organic phase, followed by stripping and electrowinning. The minor amount of extracted silver and Copper can be scrubbed from the gold-loaded organic phase before stripping. The process is suitable for obtaining the gold of $99.99 \%$ purity from aqueous cyanide solution nominally containing gold at $200 \mathrm{mg} / \mathrm{L}$, Copper $140 \mathrm{mg} / \mathrm{L}$, and silver $2 \mathrm{mg} / \mathrm{L}$. The process is also selective against nickel, cobalt, iron, and zinc, and the gold loading as high as $\mathbf{5 0 0 0}$ $\mathrm{mg} / \mathrm{L}$ can be obtained in the organic phase.

\subsection{A Review of the Hydrometallurgical Process for Recovery of Gold by Thiosulfate}

This is a review by E. Alfaro, D. Michel and J. Frenay from GEOMAC Department, University of Liege, Belgium, Ecomaterials and Ecoprocesses, Proceedings of the International Symposium on Ecomaterials and Ecoprocesses, 2nd, Vancouver, BC, Canada, Aug. 24-27, 2003 453. The thiosulfate process is presently the best non-polluting alternative to the cyanide recovery of gold. A thermodynamic and electrochemical review of the chemical system Au-S-O$\mathrm{Cu}$ as well as the distribution and the (meta) stability of ionic species and polarog.c expteriments allow a better understanding of the mechanisms of the reactions. A phenomenol. model is proposed. Important aspects such as catalytic role of $\mathrm{Cu}$, consumption of reagents (which could be important due to the metastable character of thiosulfate), reagents concentration, kinetics factors and the role of some, protective agents, such as amino acids are emphasized. The recovery of gold from pregnant solution cannot be done by active carbon; the most promising processes are based on the use of ion-exchange resins.

\section{General}

\subsection{Gold Recovery using Fabrics Coated with Conducting Polymers}

Conducting polymer coated fabrics were found to recover gold from solutions containing $\left(\mathrm{AuCl}_{4}\right]^{-}$in a facile and selective fashion. J. Ding, V. Misoska, W.E. Price, S.F. Ralph, G. Tsekouras, G.S. Wallace from the Intelligent Polymer Research Institute, University of Wollongong, Australia, Synthetic Metals 2003, 135. Although the fabric substrates also demonstrated a significant ability to recover gold, the rate of uptake by the coated fabrics was significantly faster. Conducting polymer coated fabrics also recovered gold from solutions containing $\left(\mathrm{Au}(\mathrm{CN})_{2}\right]^{-}$, however, little selectivity was observed when solutions containing both gold and copper cyanide complexes were examined. 